\title{
The Photoeffect on Graphene and Axion Detection by Graphene
}

\author{
Miroslav Pardy \\ Department of Physical Electronics \\ Masaryk University, Kotlářská , Brno \\ Czech Republic
}

\section{Introduction}

The photoelectric effect is a quantum electronic phenomenon in which electrons are emitted from matter after the absorption of energy from electromagnetic radiation. Frequency of radiation must be above a threshold frequency, which is specific to the type of surface and material. No electrons are emitted for radiation with a frequency below that of the threshold. These emitted electrons are also known as photoelectrons in this context. The photoelectric effect was theoretically explained by Einstein in his paper (Einstein, 1905), and the term "light quanta" called "photons" was introduced by chemist G. N. Lewis, in 1926. Einstein writes: In accordance with the assumption to be considered here, the energy of light ray spreading out from point source is not continuously distributed over an increasing space but consists of a finite number of energy quanta which are localized at points in space, which move without dividing, and which can only be produced and absorbed as complete units.

There is a textbook proof that the free electron in vacuum cannot absorb photon. It follows from the special theory of relativity. If $p_{1}, p_{2}$ are the initial and final 4 -momenta of electron with rest mass $m$ and $k$ is the 4-momentum of photon, then after absorption of photon by electron we write $k+p_{1}=p_{2}$, which gives when squared $k^{2}+2 k p_{1}+p_{1}^{2}=p_{2}^{2}$. Then, with $p_{1}^{2}=p_{2}^{2}=-m^{2}$ and $k^{2}=0$, we get for the rest electron with $\mathbf{p}_{1}=0$ the elementary relation $m \omega=0$, Q.E.D..

The linear dependence on the frequency was experimentally determined in 1915 when Robert Andrews Millikan showed that Einstein formula

$$
\hbar \omega=\frac{m v^{2}}{2}+A
$$

was correct. Here $\hbar \omega$ is the energy of the impinging photon, $v$ is electron velocity measured by the magnetic spectrometer and $A$ is the work function of concrete material. The work function for Aluminium is $4.3 \mathrm{eV}$, for Beryllium $5.0 \mathrm{eV}$, for Lead $4.3 \mathrm{eV}$, for Iron $4.5 \mathrm{eV}$, and so on (Rohlf, 1994). The work function concerns the surface photoelectric effect where the photon is absorbed by an electron in a band. The theoretical determination of the work function is the problem of the solid state physics. On the other hand, there is the so called atomic photoeffect (Amusia, 1987), where the ionization energy plays the role of the work function. 
The system of the ionization energies is involved in the tables of the solid state physics. The work fuction of graphene, or, work fuction of the Wigner crystal in graphene was never determined, and it is the one of the prestige problem of the contemporary experimental and theoretical graphene physics.

In case of the volume photoeffect, the ionization work function is defined in many textbooks on quantum mechanics. Or,

$$
W=\int_{x_{1}}^{x_{2}}\left(\frac{d E}{d x}\right) d x
$$

where $E$ is the energy loss of moving electron.

The formula (1) is the law of conservation of energy. The classical analogue of the equation (1) is the motion of the Robins ballistic pendulum in the resistive medium.

The Einstein ballistic principle is not valid inside of the blackbody. The Brownian motion of electrons in this cavity is caused by the repeating Compton process $\gamma+e \rightarrow \gamma+e$ and not by the ballistic collisions. The diffusion constant for electrons must be calculated from the Compton process and not from the ballistic process. The same is valid for electrons immersed into the cosmic relic photon sea.

The idea of the existence of the Compton effect is also involved in the Einstein article. He writes (Einstein, 1905): The possibility should not be excluded, however, that electrons might receive their energy only in part from the light quantum. However, Einstein was not sure, a priori, that his idea of such process is realistic. Only Compton proved the reality of the Einstein statement. The time lag between the incidence of radiation and the emission of a photoelectron is very small, less than $10^{-9}$ seconds.

At energies $\hbar \omega<W$, the photoeffect is not realized. However, the photo-conductivity is the real process for such energies. The photoeffect is realized only in medium and with low energy photons, but with energies $\hbar \omega>W$, which gives the Compton effect negligible. Compton effect can be realized with electrons in medium and also with electrons in vacuum. For $\hbar \omega \gg$ $W$ the photoeffect is negligible in comparison with the Compton effect. At the same time it is necessary to say that the Feynman diagram of the Compton effect cannot be reduced to the Feynman diagram for photoeffect. In case of the high energy gamma rays, it is possible to consider the process called photoproduction of elementary particles on protons in LHC, or photo-nuclear reactions in nuclear physics. Such processes are energetically far from the photoelectric effect in solid state physics.

Eq. (1) represents so called one-photon photoelectric effect, which is valid for very weak electromagnetic waves. At present time of the petawatt laser physics, where the high electromagnetic intensity is possible, we know that so called multiphoton photoelectric effect is possible. Then, instead of equation (1) we can write

$$
\hbar \omega_{1}+\hbar \omega_{2}+\ldots \hbar \omega_{n}=\frac{m v^{2}}{2}+A
$$

As na analogue of the equation (3), the multiphoton Compton effect is also possible: $\gamma_{1}+\gamma_{2}+$ $\ldots \gamma_{n}+e \rightarrow \gamma+e$. To our knowledge the Compton process with the entangled photons was still not discovered and elaborated. On the other hand, there is the deep inelastic Compton effect in the high energy particle physics.

More than 70 years ago, Peierls $(1934,1935)$ and Landau (1937) performed a proof that the 2-dimensional crystal is not thermodynamically stable and cannot exist. They argued that the 
thermodynamical fluctuations of such crystal lead to such displacements of atoms that these displacements are of the same size as the interatomic distances at the any finite temperature. The argument was extended by Mermin (1968) and it seemed that many experimental observations supported the Landau-Peierls-Mermin theory. So, the "impossibility" of the existence of graphene was established.

In 2004, Andre Geim, Kostia Novoselov (Novoselov et al. 2004; 2005; Kane, 2005) and co-workers at the University of Manchester in the UK by delicately cleaving a sample of graphite with sticky tape produced something that was long considered impossible: a sheet of crystalline carbon just one atom thick, known as graphene. Geim group was able to isolate graphene, and was able to visualize the new crystal using a simple optical microscope. Nevertheless, Landau-Peierls-Mermin proof is of the permanent historical and pedagogical meaning.

At present time, there are novel methods how to create graphene sheet. For instance, Dato et al. (2008) used the plasma reactor, where the graphene sheets were synthesized by passing liquid ethanol droplets into an argon plasma.

Graphene is the benzene ring $\left(\mathrm{C}_{6} \mathrm{H}_{6}\right)$ stripped out from their $\mathrm{H}$-atoms. It is allotrope of carbon because carbon can be in the crystalline form of graphite, diamond, fullerene $\left(C_{60}\right)$, carbon nanotube and glassy carbon (also called vitreous carbon).

Graphene unique properties arise from the collective behaviour of electrons. The electrons in graphene are governed by the Dirac equation. The Dirac equation in graphene physics is used for the description of so called pseudoelectrons with pseudospins formed by the hexagonal lattice.

The Dirac fermions in graphene carry one unit of electric charge and so can be manipulated using electromagnetic fields. Strong interactions between the electrons and the honeycomb lattice of carbon atoms mean that the dispersion relation is linear and given by $E=v p, v$ is called the Fermi-Dirac velocity, $p$ is momentum of a pseudoelectron.

The linear dispersion relation follows from the relativistic energy relation for small mass together with approximation that the Fermi velocity is approximately only about 300 times less than the speed of light.

The pseudospin of the pseudoelectron is constructed in the graphene physics as follows. The graphene is composed of the system of hexagonal cells and it geometrically means that graphene is composed from the systems of two equalateral triangles. If the wave function of the first triangle sublattice system is $\varphi_{1}$ and the wave function of the second triangle sublattice system is $\varphi_{2}$, then the total wave function of the electron noving in the hexagonal system is superposition $\psi=c_{1} \varphi_{1}+c_{2} \varphi_{2}$, where $c_{1}$ and are $c_{2}$ appropriate functions of coordinate $\mathbf{x}$ and functions $\varphi_{1}, \varphi_{2}$ are functions of wave vector $\mathbf{k}$ and coordinate $\mathbf{x}$. The next crucial step in the graphene physics is the definition of the new spinor function discussed by Lozovik et al.(2008).

$$
\chi=\left\{\begin{array}{l}
\varphi_{1} \\
\varphi_{2}
\end{array}\right\}
$$

and it is possible to prove that this spinor function is solution of the Pauli equation in the nonrelativistic situation and Dirac equation of the generalized case. The corresponding mass of such effective electron is proved to be zero.

The introduction of the Dirac relativistic Hamiltonian in gaphene physics is the description of the graphene physics by means of electron-hole medium. It is the analogue of the description 
of the electron-positron vacuum by the Dirac theory of quantum electrodynamics. The pseudoelectron is not an electron of QED and pseudospin is not the spin of QED, because QED is the quantum theory of the interaction of real electrons and photons where the mass of electron is the mass defined by classical mechanics and not by collective behavior in hexagonal sheet called graphene. The interaction of photons with pseudoelectrons was still not published by Geim and Novoselov and others, however, there is an analogue with the photodesintegration of deuteron being the bound state of neutron and proton. Or,

$$
\gamma+D \rightarrow n+p
$$

where dissociation energy $D$ of the process is $2.225 \mathrm{MeV}$. We use this analogy in the photoelectric effect in graphene and we must accept the idea that the initial process in the photoelectric effect in graphene is the photodesintegration of pseudoelectron and then the interaction of the photon with the real electron. So, we generalize the Einstein equation to the novel form:

$$
\hbar \omega=\frac{m v^{2}}{2}+A_{d}+A
$$

where the desintegration energy necessary for the desintegration of pseudoelectron must be determined experimentally. The today graphenic theory does not solve this problem and the corresponding experiment was not performed. We suppose that this missing experiment is crucial in the graphene physics.

After dissociation of pseudoelectron, we can use the Schrödinger equation in order to establish the photoeffect in graphene in the magnetic field. Our calculation of the photoelectric effect is applicable also for the Wigner crystal, being the crystalline phase of electrons first predicted by Eugene Wigner (1934).

We derive here the differential probability of the photoelectric effect realized at the very low temperature of graphene in the very strong magnetic field. So, the term magnetic photoeffect. We deal also with graphene on the Riemann surfaces and consider the possibility of axion detection by the graphene detectors.

\section{The quantum theory of the photoelectric effect in the 2D electron gas at zero temperature and strong magnetic field}

The photoelectric effect in graphene is presented here 100 years after the publication of well known Einstein article (Einstein, 2005). While the experimental investigation of the photoelectric effect was performed in past many times, the photoelectric effect in graphene, is still the missing experiment in the graphene physics. Many discoveries in physics were performed when the physical, or, chemical object was immersed into the magnetic field.

The photoelectric effect in semiconductor by onset of magnetic field is discussed by Kleinert et al. (2008), where the Bloch functions in a magnetic field are considered as the adequate for the solution of the problem. The calculation of photoeffect of graphene including both electron-electron and electron-photon interactions on the same footing is performed for instance by Park et al. (2010). The crucial object is the spectral function with the self-energy which accounts for the many-body interactions going beyond the mean-field picture.

Ratnikov et al. (2008a) define graphene as a two-dimensional zero gap semiconductor with zero conduction and valence band overlap to determine ground state of energy of current carriers. Then he et al. calculate the transition of graphene on a substrate to a semi-metallic 
state (Ratnikov et al. 2008b). The Ratnikov at al. theory can be applied to graphene in magnetic field and bilayer graphene in magnetic field. However, it is not immediately possible to apply the Ratnikov et al. theory to calculate the photoeffect in magnetic field. So, we use the Landau approach applied in Landau diamagnetism.

The rigorous theory of the photoeffect in the metallic thin films was given by Tamm and Schubin (1931). However, graphene is the one-atom sheet and cannot be identified with the thin film situation of Tamm and Schubin. In case of the thin metallic film, the light penetrates to the distance 1000 - 10000 atomic layers under the surface of the film, while graphene is the thin film with only one atomic layer. So, the original idea is to consider the photoeffect on the 2D electron system immersed into the constant magnetic field and graphene enables to realize this project.

The quantum mechanical description of the photoeffect is based on the appropriate S-matrix element involving the interaction of atom with the impinging photon with the simultaneous generation of the electron, the motion of which can be described approximately by the plane wave

$$
\psi_{\mathbf{q}}=\frac{1}{\sqrt{V}} e^{i \mathbf{q} \cdot \mathbf{x}}, \quad \mathbf{q}=\frac{\mathbf{p}}{\hbar},
$$

where $\mathbf{p}$ is the momentum of the ejected electron.

The standard approach consists in the definition of the cross-section by the quantum mechanical equation

$$
d \sigma=\frac{2 \pi}{\hbar}\left|V_{f i}\right| \delta\left(-I+\hbar \omega-E_{f}\right) \frac{d^{3} p}{(2 \pi)^{3}},
$$

where $I$ is the ionization energy of an atom and $E_{f}$ is the the final energy of the emitted electron, $\left|V_{f i}\right|$ is the matrix element of the transition of electron from the initial bound state to the final state. The matrix element follows from the perturbative theory and it involves the first order term of the interaction between electron and photon. We use here the Davydov elementary approach (Davydov, 1976).

We suppose here that magnetic field is applied locally to the carbon sheet, so, in a sufficient distance from it the wave function is of the form of the plane wave (7). This situation has an analog in the classical atomic effect discussed by Davydov (1976).

The probability of the emission of electron by the electromagnetic wave is of the well-known form (Davydov, 1976):

$$
d P=\frac{e^{2} p}{8 \pi^{2} \varepsilon_{0} \hbar m \omega}\left|\int e^{i(\mathbf{k}-\mathbf{q}) \cdot \mathbf{x}}(\mathbf{e} \cdot \nabla) \psi_{0} d x d y d z\right|^{2} d \Omega=C|J|^{2} d \Omega,
$$

where the interaction for absorption of the electromagnetic wave is normalized to one photon in the unit volume, $\mathbf{e}$ is the polarization of the impinging photon, $\varepsilon_{0}$ is the dielectric constant of vacuum, $\psi_{0}$ is the basic state of and atom. We have denoted the integral in $\|$ by $J$ and the constant before $\|$ by $\mathrm{C}$.

We consider the case with electrons in magnetic field as an analog of the Landau diamagnetism. So, we take the basic function $\psi_{0}$ for one electron in the lowest Landau level, as 


$$
\psi_{0}=\left(\frac{m \omega_{c}}{2 \pi \hbar}\right)^{1 / 2} \exp \left(-\frac{m \omega_{c}}{4 \hbar}\left(x^{2}+y^{2}\right)\right), \quad \omega_{c}=\frac{|e| H}{m c},
$$

which is solution of the Schrödinger equation in the magnetic field with potentials $\mathbf{A}=$ $(-H y / 2,-H x / 2,0), A_{0}=0$ (Drukarev, 1988):

$$
\left[\frac{p_{x}^{2}}{2 m}+\frac{p_{y}^{2}}{2 m}-\frac{m}{2}\left(\frac{\omega_{c}}{2}\right)^{2}\left(x^{2}+y^{2}\right)\right] \psi=E \psi .
$$

We have supposed that the motion in the z-direction is zero and it means that the wave function $\exp \left[(i / \hbar) p_{z} z\right]=1$.

So, The main problem is to calculate the integral

$$
J=\int e^{i(\mathbf{K} \cdot \mathbf{x})}(\mathbf{e} \cdot \nabla) \psi_{0} d x d y d z ; \quad \mathbf{K}=\mathbf{k}-\mathbf{q} .
$$

with the basic Landau function $\psi_{0}$ given by the equation (10).

Operator $(\hbar / i) \nabla$ is Hermitean and it means we can rewrite the last integrals as follows:

$$
J=\frac{i}{\hbar} \mathbf{e} \cdot \int\left[\left(\frac{\hbar}{i} \nabla\right) e^{i(\mathbf{K} \cdot \mathbf{x})}\right]^{*} \psi_{0} d x d y d z
$$

which gives

$$
J=i \mathbf{e} \cdot \mathbf{K} \int e^{-i(\mathbf{K} \cdot \mathbf{x})} \psi_{0} d x d y d z
$$

The integral in eq. (14) can be transformed using the cylindrical coordinates with

$$
d x d y d z=\varrho d \varrho d \varphi d z, \quad \varrho^{2}=x^{2}+y^{2}
$$

which gives for vector $\mathbf{K}$ fixed on the axis $\mathrm{z}$ with $\mathbf{K} \cdot \mathbf{x}=K z$ and with physical condition $\mathbf{e} \cdot \mathbf{k}=0$, expressing the physical situation where polarization is perpendicular to the direction of the wave propagation. So,

$$
J=(i)(\mathbf{e} \cdot \mathbf{q}) \int_{0}^{\infty} \varrho d \varrho \int_{-\infty}^{\infty} d z \int_{0}^{2 \pi} d \varphi e^{-i K z} \psi_{0}
$$

Using

$$
\psi_{0}=A \exp \left(-B Q^{2}\right) ; \quad A=\left(\frac{m \omega_{c}}{2 \pi \hbar}\right)^{1 / 2} ; \quad B=\frac{m \omega_{c}}{4 \hbar} .
$$

The integral (16) is then

$$
J=(-\pi i) \frac{A}{B}(\mathbf{e} \cdot \mathbf{q}) \int_{-\infty}^{\infty} e^{-i K z} d z=(-\pi i) \frac{A}{B}(\mathbf{e} \cdot \mathbf{q})(2 \pi) \delta(K)
$$

Then,

$$
d P=C|J|^{2} d \Omega=4 \pi^{4} \frac{A^{2}}{B^{2}} C(\mathbf{e} \cdot \mathbf{q})^{2} \delta^{2}(K) d \Omega .
$$

Now, let be the angle $\Theta$ between direction $\mathbf{k}$ and direction $\mathbf{q}$, and let be the angle $\Phi$ between planes $(\mathbf{k}, \mathbf{q})$ and $(\mathbf{e}, \mathbf{k})$. Then, 


$$
(\mathbf{e} \cdot \mathbf{q})^{2}=q^{2} \sin ^{2} \Theta \cos ^{2} \Phi
$$

So, the differential probability of the emission of photons from the graphene in the strong magnetic field is as follows:

$$
d P=\frac{4 e^{2} p}{\pi \varepsilon_{0} m^{2} \omega \omega_{c}}\left[q^{2} \cos ^{2} \Theta \sin ^{2} \Phi\right] \delta^{2}(K) d \Omega ; \quad \omega_{c}=\frac{|e| H}{m c} .
$$

We can see that our result differs form the result for the original photoelectric effect which involves the term

$$
\frac{1}{\left(1-\frac{v}{c} \cos \Theta\right)^{4}},
$$

which means that the most intensity of the classical photoeffect is in the direction of the electric vector of the electromagnetic wave $(\Phi=\pi / 2, \Theta=0)$. While the non-relativistic solution of the photoeffect in case of the Coulomb potential was performed by Stobbe (1930) and the relativistic calculation by Sauter (1931), the general magnetic photoeffect (with electrons moving in the magnetic field and forming atom) was not still performed in a such simple form. The delta term $\delta \cdot \delta$ represents the conservation law $|\mathbf{k}-\mathbf{q}|=0$ in our approximation.

\section{Photoeffect with Volkov solution}

It is valuable from the pedagogical point of view (Berestetzkii et al., 1989) to remember the Volkov solution, where the motion of the Dirac electron is considered in the following four potential

$$
A_{\mu}=a_{\mu} \varphi ; \quad \varphi=k x ; \quad k^{2}=0 .
$$

From equation (23), it follows that $F_{\mu v}=\partial_{\mu} A_{v}-\partial_{\nu} A_{\mu}=a_{v} k_{\mu}-a_{\mu} k_{v}=$ const., which means that electron moves in the constant electromagnetic field with the components $\mathbf{E}$ and $\mathbf{H}$. The parameters $a$ and $k$ can be chosen in a such a way that $\mathbf{E}=0$. So, the motion of electron is performed in the constant magnetic field.

The Volkov (1935) solution of the Dirac equation for an electron moving in a field of a plane wave was derived in the form (Berestetzkii et al., 1989; Pardy, 2003; Pardy, 2004; Pardy, 2007):

$$
\psi_{p}=\frac{u(p)}{\sqrt{2 p_{0}}}\left[1+e \frac{(\gamma k)(\gamma A(\varphi))}{2 k p}\right] \exp [(i / \hbar) S]
$$

and $S$ is an classical action of an electron moving in the potential $A(\varphi)$ :

$$
S=-p x-\int_{0}^{k x} \frac{e}{(k p)}\left[(p A)-\frac{e}{2}(A)^{2}\right] d \varphi
$$

It was shown that for the potential (23) the Volkov wave function is (Berestetzkii et al., 1989):

$$
\psi_{p}=\frac{u(p)}{\sqrt{2 p_{0}}}\left[1+e \frac{(\gamma k)(\gamma a)}{2 k p} \varphi\right] \exp [(i / \hbar) S]
$$

with 


$$
S=-e \frac{a p}{2 k p} \varphi^{2}+e^{2} \frac{a^{2}}{6 k p} \varphi^{3}-p x
$$

We used $c=\hbar=1$.

However, the relativistic wave function can be obtained by solving the Dirac equation in magnetic field. It was derived in the form (Sokolov et all. 1983).

$$
\Psi(\mathbf{x}, t)=\frac{1}{L} \exp \left\{-\frac{i}{\hbar} \epsilon E t+i k_{2} y+i k_{3} z\right\} \psi ; \quad \psi=\left(\begin{array}{c}
C_{1} u_{n-1}(\eta) \\
i C_{2} u_{n}(\eta) \\
C_{3} u_{n-1}(\eta) \\
i C_{4} u_{n}(\eta)
\end{array}\right),
$$

where $\epsilon= \pm 1$ and the spinor components are given by the following formulas:

$$
u_{n}(\eta)=\sqrt{\frac{\sqrt{2 \gamma}}{2^{n} n !} \sqrt{\pi}} e^{-\eta^{2} / 2} H_{n}(\eta)
$$

with

$$
\begin{gathered}
H_{n}(\eta)=(-1)^{n} e^{\eta^{2}}\left(\frac{d}{d \eta}\right)^{n} e^{-\eta^{2}}, \\
\eta=\sqrt{2 \gamma} x+k_{2} / \sqrt{2 \gamma} ; \quad \gamma=e H / 2 c \hbar .
\end{gathered}
$$

The coefficients $C_{i}$ are defined in the Sokolov et al. monograph (Sokolov et al., 1983). So, our approach can be generalized.

It is evident that the model which we have used is the one-pseudoelectron model of the 2D hexagon crystal. However the photoelectric effect should be considered in the many pseudoelectron model which is substantially more complicated than the one-pseudoelectron electron model. The investigation of such approach is not at present time published.

\section{Green function in magnetic field for photoeffect}

The electron propagator is a building stone in the mass operator from which can be computed the energy shift, the power spectrum of the synchrotron radiation, the anomalous magnetic moment and so on. It was intensively discussed for instance by Dittrich et al. and Schwinger et al.. Here we will follow the Dittrich treatment in order to derive the special representation of the Dirac propagator of a particle in a constant external magnetic field (Dittrich et al., 1985). The application of the formalism to graphene physics is evident.

If we write the Green function as

$$
G_{+}\left(x, x^{\prime}\right)=\left\langle x\left|G_{+}\right| x^{\prime}\right\rangle,
$$

then, from the Green function equation for spin $1 / 2$ particles

$$
(\gamma \Pi+m) G_{+}\left(x, x^{\prime}\right)=\delta\left(x-x^{\prime}\right)=\left\langle x \mid x^{\prime}\right\rangle
$$

we have

$$
G_{+}=\frac{1}{\gamma \Pi+m-i \varepsilon} ; \quad \varepsilon>0,
$$


where

$$
\Pi_{\mu}=p_{\mu}-e A_{\mu} ; \quad p_{\mu}=\frac{1}{i} \partial_{\mu}
$$

with simultaneous omitting the charge matrix $q$ in eqs. (34) and (35).

The equivalent form of $G_{+}$in eq. (34) is obviously given by relation

$$
G_{+}=\frac{\gamma \Pi-m}{(\gamma \Pi)^{2}-m^{2}-i \varepsilon}
$$

where

$$
(\gamma \Pi)^{2}=-\Pi^{2}-\frac{i}{2} \sigma_{\mu \nu}\left[\Pi_{\mu}, \Pi_{v}\right]
$$

with

$$
\sigma^{\mu v}=\frac{i}{2}\left[\gamma^{\mu}, \gamma^{v}\right], \quad\left\{\gamma^{\mu}, \gamma^{v}\right\}=-2 g^{\mu v}
$$

and

$$
\left[\Pi^{\mu}, \Pi^{v}\right]=i e F^{\mu v},
$$

which gives

$$
(\gamma \Pi)^{2}=-\Pi^{2}+\frac{e}{2} \sigma_{\mu \nu} F^{\mu \nu} .
$$

For the constant magnetic field chosen in the $z$-direction we have $F_{12}=-F_{21}=B=$ const and

$$
(\gamma \Pi)^{2}=-\Pi^{2}-e B \sigma^{12},
$$

where we designated by $\sigma^{12}$ the following matrix:

$$
\begin{aligned}
& \sigma^{12}=\left(\begin{array}{cc}
\sigma^{3} & 0 \\
0 & \sigma^{3}
\end{array}\right)= \\
& \left(\begin{array}{cccc}
1 & 0 & 0 & 0 \\
0 & -1 & 0 & 0 \\
0 & 0 & 1 & 0 \\
0 & 0 & 0 & -1
\end{array}\right) \stackrel{d}{=} \sigma^{3},
\end{aligned}
$$

where $\sigma^{3}$ is $4 \times 4$ matrix. Using the last formulas, we write

$$
G_{+}=\frac{m-\gamma \Pi}{\Pi^{2}-\kappa^{2}-i \varepsilon}
$$

where

$$
\kappa^{2} \stackrel{d}{=} m^{2}-e B \sigma^{3}
$$

Space-time representation of the Green function with potential $A$ is then

$$
G_{+}\left(x^{\prime}, x^{\prime \prime} \mid A\right)=\left\langle x^{\prime}\left|\frac{m-\gamma \Pi}{\Pi^{2}-\kappa^{2}-i \varepsilon}\right| x^{\prime \prime}\right\rangle .
$$


Now, we can evaluate the formula (45). We use the ansatz (Dittrich et al., 1985)

$$
G_{+}\left(x^{\prime}, x^{\prime \prime} \mid A\right)=\Phi\left(x^{\prime}, x^{\prime \prime}\right)\left[m-\gamma^{v}\left(\frac{1}{i} \partial_{v}^{\prime}-e A_{v}^{\prime}\left(x^{\prime}\right)\right)\right] \Delta_{+}\left(x^{\prime}, x^{\prime \prime} \mid A^{\prime}\right)
$$

with

$$
\left.A_{\mu}^{\prime}\left(x^{\prime}\right)\right]=-\frac{1}{2} F_{\mu \nu}\left(x^{\prime}-x^{\prime \prime}\right)^{v}
$$

and

$$
\Phi\left(x^{\prime}, x^{\prime \prime}\right)=\exp \left\{i e \int_{x^{\prime \prime}}^{x^{\prime}} d x_{\mu}\left[A^{\mu}(x)+\frac{1}{2} F^{\mu v}\left(x_{v}^{\prime}-x_{v}^{\prime \prime}\right)\right]\right\} .
$$

where the integral is not dependent of the choice of the integration path because of vanishing rot of the integrand. If we choose the integration path in the form of the straight line

$$
x(t)=x^{\prime \prime}+t\left(x^{\prime}-x^{\prime \prime}\right) ; \quad t \in<0,1>,
$$

we find that the second term of the integrand gives no contribution and we have instead of eq. (48):

$$
\Phi\left(x^{\prime}, x^{\prime \prime}\right)=\exp \left\{i e \int_{x^{\prime \prime}}^{x^{\prime}} d x_{\mu} A^{\mu}(x)\right\}
$$

and the derivatives are as follows:

$$
\frac{\partial \Phi\left(x^{\prime}, x^{\prime \prime}\right)}{\partial x^{\prime \mu}}=i e\left[A_{\mu}\left(x^{\prime}\right)-A_{\mu}^{\prime}\left(x^{\prime}\right)\right] \Phi\left(x^{\prime}, x^{\prime \prime}\right),
$$

where after substitution of the ansatz (46) into eq. (33) we get

$$
\left(\left(\frac{1}{i} \partial^{\prime}-e A^{\prime}\right)^{2}+\kappa^{2}\right) \Delta_{+}\left(x^{\prime}, x^{\prime \prime} \mid A\right)=\delta\left(x^{\prime}-x^{\prime \prime}\right),
$$

where $A^{\prime}$ is defined by eq. (47). After modification of eq. (52) we have

$$
\left(-\partial^{2}+\kappa^{2}-i \varepsilon-\frac{e^{2}}{4} x_{\mu} F^{2 \mu v} x_{v}\right) \Delta_{+}\left(x \mid A^{\prime}\right)=\delta(x)
$$

with

$$
F^{2 \mu \nu}=F_{\alpha}^{\mu} F^{\alpha \mu} .
$$

We can solve the equation (53) by using the Fourier transform (with the notation $(d k)=d^{4} k$ )

$$
\Delta_{+}\left(x \mid A^{\prime}\right)=\int \frac{(d k)}{(2 \pi)^{4}} \Delta_{+}\left(k \mid A^{\prime}\right)
$$

which gives

$$
\left(k^{2}+\frac{e^{2}}{4} \frac{\partial}{\partial k^{\mu}} F^{2 \mu \nu} \frac{\partial}{\partial k^{v}}+\kappa^{2}-i \varepsilon\right) \Delta_{+}\left(k \mid A^{\prime}\right)=1 .
$$

Using the ansatz 


$$
\Delta_{+}\left(x \mid A^{\prime}\right)=i \int_{0}^{\infty} d s e^{-M(i s)} e^{-i s\left(\kappa^{2}-i s\right)}
$$

with

$$
M(i s)=k^{\alpha} X_{\alpha \beta}(\text { is }) k^{\beta}+Y(\text { is }) ; \quad X_{\alpha \beta}=X_{\beta \alpha},
$$

we get from eq. (56)

$$
i \int_{0}^{\infty} d s\left\{k\left[1+e^{2} X(i s) F^{2} X(i s)\right] k-\frac{e^{2}}{2} \operatorname{tr}\left[F^{2} X\right]+\kappa^{2}-i \varepsilon\right\} e^{-M(i s)} e^{-i s\left(\kappa^{2}-i s\right)}=1,
$$

or, in the equivalent form

$$
i \int_{0}^{\infty} d s g(i s) e^{-f(i s)}=1
$$

which is equation for $X$ and $Y$.

Let us try to put

$$
g(\text { is })=f^{\prime}(\text { is })
$$

Then,

$$
i \int_{0}^{\infty} d s g(i s) e^{-f(i s)}=e^{-f(0)}-e^{-f(i \infty)} .
$$

From the comparison of eq. (62) with eq. (60) the requirement follows

$$
f(0)=0 ; \quad \operatorname{Re} f(i \infty)=\infty .
$$

The relation $g=f^{\prime}$ reads in our case

$$
k\left[1+e^{2} X(i s) F^{2} X(i s)\right] k-\frac{e^{2}}{2} \operatorname{tr}\left[F^{2} X(i s)\right]=M^{\prime}(i s) .
$$

It enables to write after rotation of the integration path according to is $\rightarrow s$, the following equations:

$$
\begin{aligned}
& 1+e^{2} X(s) F^{2}(s)=\dot{X}(s), \\
& -\frac{e^{2}}{2} \operatorname{tr}\left[F^{2} X(s)\right]=\dot{Y}(s) .
\end{aligned}
$$

The solution of eqs. (65) and (66) are

$$
\begin{aligned}
& X(s)=(e F)^{-1} \tan (e F s), \\
& Y(s)=\frac{1}{2} \operatorname{tr} \ln \cos (e F s) .
\end{aligned}
$$

which can be verified by differentiation. At the same time it can be verified the first condition in (63) is fulfilled because of $X(0)=Y(0)=0$. 
To write $X(s)$ and $Y(s)$ in the explicit form, we use the advantage of the special form of the strength tensor

$$
F^{\mu v}=\left(\begin{array}{cccc}
0 & 0 & 0 & 0 \\
0 & 0 & B & 0 \\
0 & -B & 0 & 0 \\
0 & 0 & 0 & 0
\end{array}\right)
$$

with

$$
(i F)^{2}=B^{2}\left(\begin{array}{llll}
0 & 0 & 0 & 0 \\
0 & 1 & 0 & 0 \\
0 & 0 & 1 & 0 \\
0 & 0 & 0 & 0
\end{array}\right)
$$

Then, we have:

$$
\begin{gathered}
X(i s)=(e F)^{-1} \tan (i e F s)= \\
\text { is }\left[\left(\begin{array}{llll}
1 & 0 & 0 & 0 \\
0 & 0 & 0 & 0 \\
0 & 0 & 0 & 0 \\
0 & 0 & 0 & 1
\end{array}\right)+\left(\begin{array}{llll}
0 & 0 & 0 & 0 \\
0 & 1 & 0 & 0 \\
0 & 0 & 1 & 0 \\
0 & 0 & 0 & 0
\end{array}\right) \frac{\tan (e B s)}{(e B s)}\right] .
\end{gathered}
$$

Now, let us introduce the notation for the specific vectors as follows

$$
\begin{gathered}
a_{\|} \stackrel{d}{=}\left(a^{0}, 0,0, a^{3}\right) ; \quad a_{\perp} \stackrel{d}{=}\left(0, a^{1}, a^{2}, 0\right) . \\
(a b)_{\|} \stackrel{d}{=}-a^{0} b^{0}+a^{3} b^{3} ; \quad(a b)_{\perp} \stackrel{d}{=} a^{1} b^{1}+a^{2} b^{2} .
\end{gathered}
$$

Then, $\left(X=X_{\alpha}^{\beta}\right)$

$$
k X(i s) k=i s\left[k_{\|}^{2}+\frac{\tan (e B s)}{e B s} k_{\perp}^{2}\right] .
$$

For $Y($ is $)$ we get by the similar way

$$
\begin{gathered}
Y(i s)=\frac{1}{2} \operatorname{tr} \ln \cos (i e F s)= \\
\frac{1}{2} \ln \operatorname{det}\left[\left(\begin{array}{llll}
1 & 0 & 0 & 0 \\
0 & 0 & 0 & 0 \\
0 & 0 & 0 & 0 \\
0 & 0 & 0 & 1
\end{array}\right)+\left(\begin{array}{llll}
0 & 0 & 0 & 0 \\
0 & 1 & 0 & 0 \\
0 & 0 & 1 & 0 \\
0 & 0 & 0 & 0
\end{array}\right) \cos (e B s)\right]= \\
\ln \cos (e B s) .
\end{gathered}
$$

At this stage we are prepared to compute $\exp (-f(i \infty))$. Let us recall that

$$
f(i s)=M(i s)+i s\left(\kappa^{2}-i s\right)=k X(i s) k+Y(i s)+i s\left(\kappa^{2}-i s\right) .
$$


Then,

$$
\begin{gathered}
e^{-f(i \infty)}=\lim _{s \rightarrow \infty} e^{-f(i s)}= \\
\lim _{s \rightarrow \infty} \exp \left\{-i s\left(k_{\|}^{2}+k_{\perp}^{2} \frac{\tan (e B s)}{e B s}\right)\right\} \frac{e^{-i s\left(\kappa^{2}-i s\right)}}{\cos (e B s)}=0 .
\end{gathered}
$$

If we introduce

$$
z=e B s
$$

we get from eqs. (55), (57), and (75)

$$
\begin{gathered}
\Delta\left(x^{\prime}, x^{\prime \prime} \mid A\right)=i \int_{0}^{\infty} d s \Phi\left(x^{\prime}, x^{\prime \prime}\right) \int \frac{(d k)}{(2 \pi)^{4}} e^{i k\left(x^{\prime}-x^{\prime \prime}\right)} \times \\
\frac{1}{\cos z} \exp \left\{-i s\left(k_{\|}^{2}+k_{\perp}^{2} \frac{\tan (z)}{z}\right)\right\} e^{-i s\left(\kappa^{2}-i s\right)} .
\end{gathered}
$$

However, because of

$$
\Delta_{+}\left(x^{\prime}, x^{\prime \prime} \mid A\right)=\left\langle x^{\prime}\left|\frac{1}{\Pi^{2}+\kappa^{2}-i \varepsilon}\right| x^{\prime \prime}\right\rangle=i \int_{0}^{\infty} d s\left\langle x^{\prime}\left|e^{-i s \Pi^{2}}\right| x^{\prime \prime}\right\rangle e^{-i s\left(\kappa^{2}-i s\right)}
$$

we get after comparison of eq. (79) with eq. (78)

$$
\left\langle x^{\prime}\left|e^{-i s \Pi^{2}}\right| x^{\prime \prime}\right\rangle=\Phi\left(x^{\prime}, x^{\prime \prime}\right) \int \frac{(d k)}{(2 \pi)^{4}} e^{i k\left(x^{\prime}-x^{\prime \prime}\right)} \frac{1}{\cos z} e^{-i s\left(k_{\|}^{2}+k_{\perp}^{2} \frac{\tan (z)}{z}\right)} .
$$

The formula (80) may be used as a starting point in derivation of the further formulas. For instance, putting $s \rightarrow a_{\perp} s$ in eq. (80) we get

$$
\begin{gathered}
\left\langle x^{\prime}\left|e^{-i s a_{\perp} \Pi^{2}}\right| x^{\prime \prime}\right\rangle= \\
\Phi\left(x^{\prime}, x^{\prime \prime}\right) \int \frac{(d k)}{(2 \pi)^{4}} e^{i k\left(x^{\prime}-x^{\prime \prime}\right)} \frac{1}{\cos \left(a_{\perp} z\right.} \exp \left\{-i s\left(k_{\|}^{2}+k_{\perp}^{2} \frac{\tan \left(a_{\perp} z\right)}{a_{\perp} z}\right) a_{\perp}\right\} .
\end{gathered}
$$

Further, because of

$$
\left\langle x^{\prime}\left|e^{-i s a_{\perp} \Pi^{2}} \Pi_{\mu}\right| x^{\prime \prime}\right\rangle=\left(i \partial^{\prime \prime}-e A\left(x^{\prime \prime}\right)\right)_{\mu}\left\langle x^{\prime}\left|e^{-i s a_{\perp} \Pi^{2}}\right| x^{\prime \prime}\right\rangle
$$

we get

$$
\begin{gathered}
\left\langle x^{\prime}\left|\exp \left\{-i s\left(a^{0} \Pi_{0} \Pi^{0}+a^{3} \Pi_{3} \Pi^{3}+a_{\perp} \Pi_{\perp}^{2}\right)\right\}\right| x^{\prime \prime}\right\rangle= \\
\left\langle x^{\prime}\left|e^{-i s a_{\perp} \Pi^{2}} \exp \left\{-i s\left(\left(a^{0}-a_{\perp}\right) \Pi_{0} \Pi^{0}+\left(a^{3}-a_{\perp}\right) \Pi_{3} \Pi^{3}\right)\right\}\right| x^{\prime \prime}\right\rangle= \\
\exp \left\{-i s\left(-\left(a^{0}-a_{\perp}\right)\left(i \partial^{\prime \prime 0}-e A^{0}\left(x^{\prime \prime}\right)\right)^{2}+\left(a^{3}-a_{\perp}\right)\left(i \partial^{\prime \prime 3}-e A^{3}\left(x^{\prime \prime}\right)\right)^{2}\right)\right\} \\
\times\left\langle x^{\prime}\left|e^{-i s a_{\perp} \Pi^{2}}\right| x^{\prime \prime}\right\rangle .
\end{gathered}
$$


Using

$$
\partial^{\prime \prime \mu} \Phi\left(x^{\prime}, x^{\prime \prime}\right)=-i e\left(A^{\mu}\left(x^{\prime \prime}\right)-\frac{1}{2} F^{\mu v}\left(x^{\prime}-x^{\prime \prime}\right) v\right) \Phi\left(x^{\prime}, x^{\prime \prime}\right)
$$

and

$$
\begin{gathered}
\exp \left\{-i s\left(-\left(a^{0}-a_{\perp}\right)\left(i \partial^{\prime \prime \prime}\right)^{2}+\left(a^{3}-a_{\perp}\right)\left(i \partial^{\prime \prime 3}\right)^{2}\right)\right\} e^{i k\left(x^{\prime}-x^{\prime \prime}\right)}= \\
\exp \left\{-i s\left(-\left(a^{0}-a_{\perp}\right)\left(k^{0}\right)^{2}+\left(a^{3}-a_{\perp}\right)\left(k^{3}\right)^{2}\right)\right\} e^{i k\left(x^{\prime}-x^{\prime \prime}\right),}
\end{gathered}
$$

we get from eqs. (82) and (83)

$$
\begin{gathered}
\left\langle x^{\prime}\left|\exp \left\{-i s\left(a^{0} \Pi_{0} \Pi^{0}+a^{3} \Pi_{3} \Pi^{3}+a_{\perp} \Pi_{\perp}^{2}\right)\right\} \Pi^{\mu}\right| x^{\prime \prime}\right\rangle= \\
\Phi\left(x^{\prime}, x^{\prime \prime}\right) \int \frac{(d k)}{(2 \pi)^{4}} e^{i k\left(x^{\prime}-x^{\prime \prime}\right)} \times \\
\frac{1}{\cos \left(a_{\perp} z\right)} \exp \left\{-i s\left(a^{0} k_{0} k^{0}+a^{3} k_{3} k^{3}+a_{\perp} k_{\perp}^{2} \frac{\tan \left(a_{\perp}\right) z}{a_{\perp} z}\right)\right\} .
\end{gathered}
$$

Similarly, we can derive

$$
\begin{gathered}
\left\langle x^{\prime}\left|\exp \left\{-i s\left(a^{0} \Pi_{0} \Pi^{0}+a^{3} \Pi_{3} \Pi^{3}+a_{\perp} \Pi_{\perp}^{2}\right)\right\}\right| x^{\prime \prime}\right\rangle= \\
\Phi\left(x^{\prime}, x^{\prime \prime}\right) \int \frac{(d k)}{(2 \pi)^{4}} e^{i k\left(x^{\prime}-x^{\prime \prime}\right)} \frac{1}{\cos \left(a_{\perp} z\right)}\left[k^{\mu}-e a_{\perp} s F^{\mu v} k_{\nu} \frac{\tan \left(a_{\perp} z\right)}{a_{\perp} z}\right] \times \\
\exp \left\{-i s\left(a^{0} k_{0} k^{0}+a^{3} k_{3} k^{3}+a_{\perp} k_{\perp}^{2} \frac{\tan \left(a_{\perp}\right) z}{a_{\perp} z}\right)\right\} .
\end{gathered}
$$

From this equation and using

$$
\gamma_{\mu} F^{\mu v} k_{\nu} \tan \left(a_{\perp} z\right)=\left(\gamma_{1} k_{2}-\gamma_{2} k_{1}\right) B \tan \left(a_{\perp} z\right)=\frac{i}{\cos \left(a_{\perp} z\right)} B \sin \left(a_{\perp} z \sigma^{3}\right)(\gamma k)_{\perp}
$$

and

$$
(k \gamma)_{\perp}-e s a_{\perp}\left(\gamma_{\mu} F^{\mu v} k_{v}\right) \frac{\tan \left(a_{\perp} z\right)}{a_{\perp} z}=\frac{1}{\cos \left(a_{\perp} z\right)} e^{-i a_{\perp} z \sigma^{3}}(\gamma k)_{\perp}
$$

we get the following relations

$$
\begin{gathered}
\left\langle x^{\prime}\left|\exp \left\{-i s\left(a^{0} \Pi_{0} \Pi^{0}+a^{3} \Pi_{3} \Pi^{3}+a_{\perp} \Pi_{\perp}^{2}\right)\right\}\left(1, \gamma^{0} \Pi^{0}, \gamma^{3} \Pi^{3},(\gamma \Pi)_{\perp}\right)\right| x^{\prime \prime}\right\rangle= \\
\Phi\left(x^{\prime}, x^{\prime \prime}\right) \int \frac{(d k)}{(2 \pi)^{4}} e^{i k\left(x^{\prime}-x^{\prime \prime}\right)} \frac{1}{\cos \left(e B s a_{\perp} z\right)} \times \\
\exp \left\{-i s\left(a^{0} k_{0} k^{0}+a^{3} k_{3} k^{3}+a_{\perp} k_{\perp}^{2} \frac{\tan \left(e B s a_{\perp}\right)}{e B s a_{\perp}} k_{\perp}^{2}\right)\right\} \times
\end{gathered}
$$




$$
\left(1, \gamma^{0} k^{0}, \gamma_{3} k^{3}, \frac{1}{\cos \left(e B s a_{\perp}\right)} e^{-i e B s a_{\perp} \sigma^{3}}(\gamma k)_{\perp}\right)
$$

For the propagator $G_{+}\left(x^{\prime}, x^{\prime \prime}\right)$ which we write in the form

$$
\begin{gathered}
G_{+}\left(x^{\prime}, x^{\prime}\right)=\left\langle x^{\prime}\left|\frac{m-\gamma \Pi}{\Pi^{2}-\kappa^{2}-i \varepsilon}\right| x^{\prime \prime}\right\rangle= \\
i \int_{0}^{\infty} d s e^{-i s\left(\kappa^{2}-i s\right)}\left\langle x^{\prime}\left|e^{-i s \Pi^{2}}(m-\gamma \Pi)\right| x^{\prime \prime}\right\rangle,
\end{gathered}
$$

we get

$$
G_{+}\left(x^{\prime}, x^{\prime}\right)=\Phi\left(x^{\prime}, x^{\prime \prime}\right) \int \frac{(d k)}{(2 \pi)^{4}} e^{i k\left(x^{\prime}-x^{\prime \prime}\right)} G_{+}(k)
$$

where

$$
\begin{aligned}
G(k)=i \int_{0}^{\infty} & d s \exp \left\{-i s\left(m^{2}-i \varepsilon+k_{\|}^{2}+\frac{\tan z}{z} k_{\perp}^{2}\right)\right\} \times \\
& \frac{e^{i \sigma^{3} z}}{\cos z}\left(m-\gamma k_{\|}-\frac{e^{-i \sigma^{3} z}}{\cos z} \gamma k_{\perp}\right) .
\end{aligned}
$$

This representation of the Green function of electron in the constant magnetic field will be used in calculation of the mass operator and the polarization tensor in the constant magnetic field.

The mass operator tin the $x$-representation is usually denoted by the symbol $M\left(x^{\prime}, x^{\prime \prime}\right)$ and in the past literature as $\Sigma\left(x^{\prime}, x^{\prime \prime}\right)$. It is defined by the relation (Schvinger, 1988):

$$
M\left(x^{\prime}, x^{\prime \prime}\right)=i e^{2} \gamma^{\mu} G_{+}\left(x^{\prime}, x^{\prime \prime}\right) D_{+}\left(x^{\prime}-x^{\prime \prime}\right) \gamma_{\mu}+\text { C.T., }
$$

where $D_{+}\left(x^{\prime}-x^{\prime \prime}\right)$ is the photon propagator

$$
D_{+}(x)=\int \frac{(d k)}{(2 \pi)^{4}} \frac{e^{i k x}}{k^{2}-i \varepsilon}
$$

and $G_{+}\left(x^{\prime}, x^{\prime \prime}\right)$ is the electron propagator

$$
G_{+}\left(x^{\prime}, x^{\prime \prime}\right)=\Phi\left(x^{\prime}, x^{\prime \prime}\right) \int \frac{(d p)}{(2 \pi)^{4}} e^{i p\left(x^{\prime}-x^{\prime \prime}\right)} G(p),
$$

where $\Phi\left(x^{\prime}, x^{\prime \prime}\right)$ and $G(p)$ are function which were specified.

The contact terms C.T. in eq. (94) can be determined by the physical normalization condition such that for $\gamma \Pi \rightarrow-m$, both $M$ and its first derivative with respect to $\gamma \Pi$ are zero. The motivation for such definition of the contact terms can be found in the monography of Dittrich and Reuter (1985).

The considered formalism can be reduced to the two-dimensional situation which is the situation of the graphene physics. The two-dimensional Green function applied to the dynamical mass generation was elaborated by Shpagin (1996) and can be also applied to the graphene physics. 


\section{Axion detection by graphene}

Axions were proposed as an extension to the Standard Model of particle physics to explain why $\mathrm{CP}$ violation, it is charge-parity symmetry violation, is observed in weak but not strong interactions - the so-called strong-CP problem.

The CP violation is measured in terms of a parameter denoted usually by symbol $\theta$ in QCD and also in Glashow-Weinberg-Salam electroweak model (GWS) and experimentally is observed that

$$
\left.|\theta|=\left|\theta_{Q C D}+\theta_{G W S}\right|<10\right)^{-9} \approx 0,
$$

which means that the strong interactions are invariant with regard to the $\mathrm{CP}$ transformation. The question is why $\theta$ is so small?

One of the most striking consequences of $\mathrm{CP}$-violation is the calculated neutron electric dipole moment $(E D M)$ to be ten orders of magnitude larger than its measured upper limit.

The total EDM is expected to receive contributions from both the $\mathrm{TeV}$ electroweak scale, via the quark spin, and from the GeV QCD scale, via the spatial distribution of the quark wave function within the neutron. It is difficult to understand how this two contributions could cancel to such precision to produce a $\mathrm{CP}$ conserving QCD ground state without fine tuning of parameters. Or, in other words, why $\mathrm{A}+\mathrm{B}=0$, if $\mathrm{A}$ and $\mathrm{B}$ have different physical origin.

The problem was solved by Peccei, Quinn, Weinberg and Wilczek by introducing a new scalar field which rolls within its potential into a state of minimum action, a CP conserving QCD vacuum state. Any imbalance between the contributions to the EDM of neutron from $\mathrm{TeV}$ and $\mathrm{GeV}$ scales is absorbed by an axion. Axion is a postulated particle which is generated by the Higgs mechanism.

The Higgs mechanism as the mechanism of the spontaneous broken symmetry can be demonstrated easily by the Lagrangian for the scalar field $\varphi$ (Kane, 1987):

$$
L=T-V=\frac{1}{2} \partial_{\nu} \varphi \partial^{v} \varphi-\left(\frac{1}{2} \mu^{2} \varphi^{2}+\frac{1}{4} \lambda \varphi^{4}\right) \ldots,
$$

where $\mu, \lambda$ are some constants.

The potential energy has its minimum for $\mu^{2}<0$ at points

$$
\varphi_{\min }= \pm \sqrt{-\frac{\mu^{2}}{\lambda}} \equiv \pm v
$$

We can write around the minimal point that

$$
\varphi(x)=v+\eta(x) .
$$

Then after insertion of eq. (100) into the original Lagrangian (98) we get approximately

$$
L \approx \frac{1}{2} \partial_{\nu} \varphi \partial^{v} \varphi-\left(\lambda v^{2} \eta^{2}+\lambda v \eta^{2}+\frac{1}{2} \mu^{2} \varphi^{2}+\frac{1}{4} \lambda \eta^{4}\right)+\ldots \quad .
$$

It is elementary to see that the mass term in the last Lagrangian is the mass term of the field $\eta$ and it is:

$$
m_{\eta}^{2}=2 \lambda v^{2}=-2 \mu^{2}>0
$$


In case of the axionic situation the mass which is generated by he so called Higgs mechanism just described, is the mass of axion.

The interaction of axionic field $a$ with the electromagnetic field is postulate in the literature as

$$
L_{\text {inter }} \approx g a \mathbf{E} \cdot \mathbf{B},
$$

where $g$ is some interaction constant of axionic field with the electric and magnetic field E, $\mathbf{B}$. In case of the interaction of axion with the electron via the Compton process, the interaction Lagrangian is postulate as follows:

$$
L_{a e}=\frac{\text { const }}{2 m_{e}}\left(\bar{\psi} \gamma^{\mu} \gamma^{5} \psi\right) \partial_{\mu} a .
$$

In case that the Compton process is considered in the external electromagnetic field, the $\psi$ functions must be determined from the Dirac equation involving the external electromagnetic field.

In case of the interaction of the axion with the graphene, the interaction is the axion-pseudoelectron interaction via the Compton process, then the wave function $\psi$ is the wave function of the graphene pseudoelectron. Graphene is considered in the presence of no external field, or in the presence of the external electromagnetic field. This process was still not solved in graphene physics, or in the physics of elementary particles.

The candidate for source of axions is considered in the last years the Sun. A decade ago US Brookhaven Laboratory first pointed an axion telescope at the Sun - a highly useful source of weakly interacting particles for fundamental research.

Axions would be produced in the Sun through the scattering of photons from electric charges the Primakoff effect - and their numbers could equal those of solar neutrinos. The idea behind the Brookhaven experiment, first proposed by Pierre Sikivie, was to put the Primakoff effect to work in reverse, using a magnetic field to catalyze conversion of solar axions back into X-ray photons of a few kilo-electronvolts.

The CERN Solar Axion Telescope, CAST, tries the detection of axions originating from the 15 million degree plasma in the Sun's core.

There is also the alternative look on axion as an physical object existing only inside of a medium and not as a free elementary particle. Then, the detection of free axion is not possible. The analogue of this situation is for instance the Cooper pair which is present only inside of superconductor and not outside of superconductor.

\section{Photoeffect on graphene wrapped on the geometrical surface}

Carbon hexagonal structures can be formed also on surfaces and not only on the Euclidean sheet. We first determine the density of states of graphene from QFT. The density of states is the imaginary part $\Im G$ of the Green function integrated over all positions, in the limit $\mathbf{x} \rightarrow \mathbf{x}^{\prime}$ (Cortijo, et al., 2007a).

$$
\varrho(\omega)=\int \Im G(\omega, \mathbf{x}, \mathbf{x}) d \mathbf{x} .
$$

In terms of the Green function in momentum representation, $\varrho(\omega)$ can be written as:

$$
\varrho(\omega)=\Im \iint \frac{d \mathbf{k}}{(2 \pi)^{2}} \frac{d \mathbf{k}^{\prime}}{(2 \pi)^{2}} e^{i \mathbf{k} \mathbf{x}} e^{i \mathbf{k}^{\prime} \mathbf{x}} G\left(\omega, \mathbf{k}, \mathbf{k}^{\prime}\right) d \mathbf{x} .
$$


The integration over $\mathbf{x}$ gives delta function $4 \pi^{2} \delta\left(\mathbf{k}-\mathbf{k}^{\prime}\right)$, and $\varrho(\omega)$ then reads:

$$
\varrho(\omega)=\int \frac{d \mathbf{k}}{(2 \pi)^{2}} \Im G(\omega, \mathbf{k}, \mathbf{k}) .
$$

Generalization to the curvature is possible by including the local curvature coupling of the Dirac equation to a curved space. This approach was historically introduced to study the electronic spectrum of closed fullerenes and has been used for fullerenes of different shapes. In case of a two dimensional space in presence of a single cosmic string in polar coordinates, the metric is (Cortijo et al., 2007a):

$$
-d s^{2}=-d t^{2}+d r^{2}+c^{2} r^{2} d \theta^{2}
$$

where the parameter $\mathrm{c}$ is a constant related to the deficit angle by $c=1-b$.

The dynamics of a massless Dirac spinor in a curved spacetime is governed by the Dirac equation:

$$
i \gamma^{\mu} \nabla_{\mu} \psi=0 .
$$

The difference with the flat space lies in the definition of the $\gamma$ matrices that satisfy generalized anti-commutation relations

$$
\left\{\gamma^{\mu}, \gamma^{\nu}\right\}=2 g^{\mu \nu}
$$

where $g^{\mu \nu}$ is given by (108), and in the covariant derivative operator, defined as

$$
\nabla_{\mu}=\partial_{\mu}-\Gamma_{\mu},
$$

where $\Gamma_{\mu}$ is the spin connection of the spinor field that can be calculated using the tetrad formalism (Cortijo et al., 2007a).

From equation (109), we can write down the Dirac equation for the electron propagator, $S_{F}\left(x, x^{\prime}\right)$ :

$$
i \gamma^{\mu}(x)\left(\partial_{\mu}-\Gamma_{\mu}\right) S_{F}\left(x, x^{\prime}\right)=\frac{1}{\sqrt{-g}} \delta^{3}\left(x-x^{\prime}\right),
$$

where $\mathbf{x}=(\mathbf{t}, \mathbf{x})$. The local density of states $N(\omega, \mathbf{x})$ is obtained by Fourier transforming the time component and taking the limit $\mathbf{x} \rightarrow \mathbf{x}^{\prime}$ :

$$
N(\omega, \mathbf{x})=\Im \operatorname{Tr} S_{F}(\omega, \mathbf{x}, \mathbf{x}) .
$$

The solution was performed considering the curvature induced by the defects as a perturbation of the flat graphene layer. The details of the calculation is given elsewhere (Cortijo et al., 2007b )

The ideas considered here can be extended to the general Riemann metric. However, the Riemann metric is also the metric of general relativity. So, there is the analogy between metric generated by deformation of the Euclidean surface and Einstein gravity, which is the Tartaglia et al. (2009) and author idea (Pardy, 2005). The deformed graphene obviously leads to the modification of the photoeffect in graphene and it can be used as the introduction to the photoelectric effect influenced by the gravitational field. 
The generalization of our formalism to the Lobačcevskii geometry is also possible if we consider the Lobačevskii geometry as the partial sum of the local pseudospheres, where pseudosphere is the two dimensional manifold with the constant negative curvature. It was proved by Beltrami (Mc Cleary, 1994), that the Lobačevskii geometry is the geometry of the sphere with the imaginary radius (iR), where $\mathrm{R}$ is the radius of sphere.

\section{Discussion}

The article is in a some sense the preamble to the any conferences of ideas related to the photoeffect on graphene, bi-layer graphite, n-layer graphite and on the Wigner crystals which are spontaneously formed in graphite structures, or, in other structures. We have considered the photoeffect on the planar crystal at zero temperature and in the very strong magnetic field. We calculated only the process which can be approximated by the Schrödinger equation for an electron orbiting in magnetic field.

At present time, the most attention in graphene physics is devoted to the conductivity of a graphene with the goal to invent new MOSFETs and new transistors for new computers forming the basis for the artificial intelligence. However, we do not know, a priori, how many discoveries are involved in the investigation of he photo-electric effects in graphene.

An pseudoelectron in the graphene is considered as the particle with all attributes of electron as an elementary particle and it is the consequence of the collective quantum motion of crystal electrons described by the solid state physics.

The existence of the pseudoelectron in vacuum is not possible. The photoeffect on graphene is the process where the photon interacts with the pseudoelectron and after some time the pseudoelectron decays in such a way that the decay product is electron. At present time the theory of such process was not elaborated. The new experiments are necessary in order to verify the photoelectric equation in graphene.

The ballistic interaction of photon with electron is not possible in vacuum for point-like electrons. The experiments in CERN, Hamburg, Orsay, (The L3 collaboration, CELLO collaboration, ALEPH collaboration, ...) and other laboratories confirmed that there are no excited states of electron in vacuum. In other words, ballistic process in vacuum with electrons was not confirmed. It means that electron in the standard model of elementary particles is a point particle. It seems that the ballistic process is possible only in a medium. However, not only in the photoelectric media. We know, that electron accelerated in PASER by the ballistic method is accelerated for instance in $\mathrm{CO}_{2}$. On the other hand, the photo-desintegrations of nuclei involves the ballistic process (Levinger, 1960), or, the interaction of light with carbon $C_{60}, C_{70}, C_{80} \ldots$ involves also the ballistic process with photons. The interaction of photon with pseudolectron in graphene is also the ballistic process.

The photoelectric effect at zero temperature can be realized only by very short laser pulses, because in case of the continual laser irradiation the zero temperature state is not stable. Only very short pulses can conserve the zero temperature $2 \mathrm{D}$ of the system. So, the experiment needs the laser with very short laser pulses.

The interaction of axion with pseudoelectron is also considered here, however this process is substantially new in the physics of elementary particles. The axion detection by graphene is based on the interaction of axion with pseudoelectron.

The information on the photoelectric effect in graphene and also the elementary particle interaction with graphene is necessary not only in the solid state physics, but also in the 
elementary particle physics in the big laboratories where graphene can form the substantial components of the particle detectors. The graphene can be probably used as the appropriate components in the solar elements, the cathode surface in the electron microscope, or, the medium of the memory hard disks in the computers. We hope that these possibilities will be consider in the physical laboratories.

The Volkov solution of the Dirac equation in the magnetic field was also mentioned. Such solution was used by Ritus (1979), Nikishov (1979) and others. Author (Pardy, 2003, 2004, 2007), used it for the electron in the laser field, synchrotron radiation, or, in case of the massive photons leading to the Riccati equation (Pardy, 2004).

We did not consider the photoeffect in LHC where the orbiting protons can be irradiated by the laser gun if installed in the tube of LHC. The elaboration of such difficult problem needs specific approach to the problem.

The graphene can be deformed in such a way that the metric of the deformed sheet is the Riemann one (Cortijo, et al., 2007b). However, the Riemann metric of general relativity is the metric of the deformed 4D sheet as was proposed by Tartaglia et al. (2009). So, in other words, there is the analogy between deformation and Einstein gravity (Pardy, 2005). The deformed graphene obviously leads to the modification of the photoeffect in graphene leading to the theory of the photoelectric effect influenced by the gravitational field.

The monolithic structures can be also built into graphene by addition and re-arrangement of carbon atoms (Lusk et al., 2008). The repeating patterns can be created to form new carbon allotropes called haeckelites. The introducing such architectonic defects modifies mechanical, electrical and chemical properties of graphene. The unconventional materials can be prepared by such technique in order to do revolution in the solid state physics.

While the last century economy growth was based on the inventions in the Edison-Tesla electricity, the economy growth in this century will be obviously based on the graphene physics. We hope that these perspective ideas will be considered at the universities and in the physical laboratories.

\section{References}

Amusia, M. Y. (1987). Atomic photoeffect, (Moscow, Nauka). (in Russian).

Berestetzkii, V. B.; Lifshitz, E. M. and Pitaevskii, L. P. (1989). Quantum electrodynamics (Moscow, Nauka). (in Russian).

Cortijo, A. and Vozmediano, M. A. H. (2007a). Electronic properties of curved graphene sheet, Europhys. Lett. (EPL) 77,47002.

Cortijo, A. and Vozmediano, M. A. H, (2007b). Nucl. Phys. B 763, 293.

Dato, A.; Radmilovic, V.; Lee, Z.; Philips, J. and Frenklach, M. (2008). Substrate-free gas phase synthesis of graphene sheets, Nano Lett., 8 (7), 2012.

Davydov, A. S. (1976). Quantum mechanics (Pergamon press, Oxford, ....).

Dittrich, W. and Reuter, M. (1985). Effective Lagrangians in Quantum Electrodynamics, Lecture Notes in Physics 220, (Springer-Verlag, Berlin, Heidelberg, New York, Tokyo).

Drukarev, G. F. (1988). Quantum mechanics (St. Petersburgh University). (in Russsian).

Einstein, A. (1905). Über einen die Erzeugung und Verwandlung des Lichtes betreffenden heuristischen Gesichtspunkt, Annalen der Physik, 17, 132. (in German); There is an 
English translation: On the Heuristic Viewpoint Concerning the Production and Transformation of Light, in AJP, 33, No. 5, May (1965).

Kane, Ch. L. (2005). Erasing electron mass, Nature, 438, November 205, 168.

Kane, G. (1987). Modern Elementary particle physics (Addison-Wesley Publishing Company, Inc.).

Kleinert, H. and Xue She-S. (2008). Photoproduction in semiconductors by onset of magnetic field, arXiv:0810.2367v1 [cond-mat.other].

Landau, L. D. (1937). Zur Teorie der Phasenumwandlungen II., Phys. Z. Sowjetunion 11, 26.

Levinger, J. S. (1960). Nuclear photo-desintegration, (Oxford University Press). (in Russian).

Lozovik, Yu. E.; Merkulov, S. P. and Sokolik, A. A. (2008). Collective electron phenomena in graphene, Uspekhi Fiz. Nauk, 178, (7), 758.

Lusk, M. T.; Carr, L. D. (2008). cond-mat.mtrl-sci/0809.3160v2. Creation of graphene allotropes using patterned defects.

Mc Cleary, J. (1994).Geometry from a differentiable viewpoint, (Cambridge University Press).

Mermin, N. D. (1968). Crystalline order in two dimensions, Phys. Rev., 176.

Nikishov, A. I. (1979). The problem of the intensive external field in quantum electrodynamics Trudy FIAN 111, 152. (in Russian).

Novoselov, K. S.; Geim, A.K.; Morozov, S. V.; Jiang, D.; Zhang. Y.; Dubonos, S. V.; Grigorieva, I.V. and Firsov, A.A. (2004). Electric field effect in atomically thin Carbon films, Science, 306, 666-669.

Novoselov, K.S.; Geim, A. K.; Morozov, S.V. et al. (2005). Two-dimensional gas of massless Dirac fermions in graphene, Nature, 438, 197.

Pardy, M. (2003). Electron in the ultrashort laser pulse, Int. J. Theor. Phys., 42(1), 99, 127.

Pardy, M. (2004). Massive photons and the Volkov solution, International Journal of Theoretical Physics, 43(1), 127.

Pardy, M. (2005). Light in metric space-time and its deflection by the screw dislocation, in: Spacetime Physics Research Trends. Horizons in World Physics, Volume 248, ISBN 1-59454-322-4, 111-125.

Pardy, M. (2007). The synchrotron radiation from the Volkov solution of the Dirac equation, arXiv: hep-ph/0703102v1.

Park, C.; Gustino, F.; Spataru, C. D.; Cohen, M.L. and Louie, S. G. (2010). Angle resolved photoemission spectra of graphene from first-principles calculations, arXiv: 1001.2803v1 [cond-mat.mtrl-sci].

Peierls, R. E. (1934). Bemerkungen über Umwandlungstemperaturen. Helvetica Physica Acta 7, 81; ibid: (1935). Quelques proprietes typiques des corpses solides. Ann. Inst. Henri Poincaré 5, 177.

Ratnikov, P. V. and Silin, A. P. (2008a). Ground state energy of current carriers in graphene, arXiv: 0801. 4642v1. arXiv: 0801.4642v1 [condmat. other].

Ratnikov. P. V. and Silin, A. P. (2008b). Transition of graphene on a substrate to a semimetallic state, arXiv: 0805.4510v1 [cond-mat.other].

Ritus, V. I. (1979). The quantum effects of the interaction of elementary particles with the intense electromagnetic field, Trudy FIAN 111, 5. (in Russian).

Rohlf, J. W. (1994). Modern Physics from $\alpha$ to $Z^{0}$ (John Willey \& Sons, Inc., New York).

Sauter, F. (1931). Über atomaren Photoeffekt bei Grosser Härte der Anwegenden Strahlung, Ann. der Phys. 9, 217. 
Sokolov, A. A. and Ternov, I. M. (1983). Relativistic electron, 2nd ed., (Moscow, Nauka). (in Russian).

Schwinger, J. (1988). Particles, Sources and Fields, (Addison-Wesley Publishing Company, Inc.).

Shpagin, A. V. (1996). Dynamical mass generation in 2+1-dimensional electrodynamics in an external magnetic field, arXiv: hep-ph/9611412.

Stobbe, M. (1930). Zur Quantenmechanik photoelektrischer Prozesse, Ann. der Phys. 7, 661.

Tamm, I. E.; Schubin, S. P. (1931). Zur Theorie des Photoeffektes an Metallen, Zeitschrift für Physik 131, 68, 97.

Volkov, D. M. (1935). Über eine Klasse von Lösungen der Diracschen Gleichung, Zeitschrift für Physik 94, 250.

Tartaglia, A. and Radicella, N. (2009). From Elastic Continua to Space-time, arXiv: 0911.3362v1 [gr-qc].

Wigner, E. P. (1935). On the Interaction of Electrons in Metals, Phys. Rev. 46, 1002. 


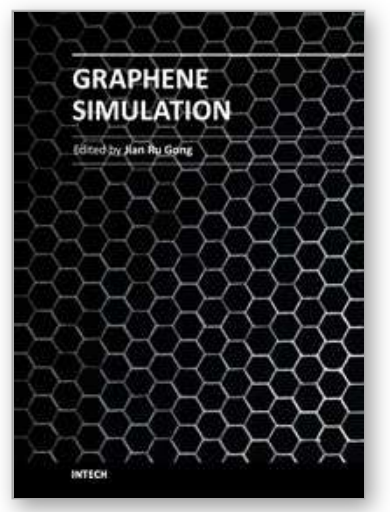

\author{
Graphene Simulation \\ Edited by Prof. Jian Gong
}

ISBN 978-953-307-556-3

Hard cover, 376 pages

Publisher InTech

Published online 01, August, 2011

Published in print edition August, 2011

Graphene, a conceptually new class of materials in condensed-matter physics, has been the interest of many theoretical studies due to the extraordinary thermal, mechanical and electrical properties for a long time. This book is a collection of the recent theoretical work on graphene from many experts, and will help readers to have a thorough and deep understanding in this fast developing field.

\title{
How to reference
}

In order to correctly reference this scholarly work, feel free to copy and paste the following:

Miroslav Pardy (2011). The Photoeffect on Graphene and Axion Detection by Graphene, Graphene Simulation, Prof. Jian Gong (Ed.), ISBN: 978-953-307-556-3, InTech, Available from:

http://www.intechopen.com/books/graphene-simulation/the-photoeffect-on-graphene-and-axion-detection-bygraphene

\section{INTECH}

open science | open minds

\author{
InTech Europe \\ University Campus STeP Ri \\ Slavka Krautzeka 83/A \\ 51000 Rijeka, Croatia \\ Phone: +385 (51) 770447 \\ Fax: +385 (51) 686166 \\ www.intechopen.com
}

\author{
InTech China \\ Unit 405, Office Block, Hotel Equatorial Shanghai \\ No.65, Yan An Road (West), Shanghai, 200040, China \\ 中国上海市延安西路65号上海国际贵都大饭店办公楼 405 单元 \\ Phone: +86-21-62489820 \\ Fax: $+86-21-62489821$
}


(C) 2011 The Author(s). Licensee IntechOpen. This chapter is distributed under the terms of the Creative Commons Attribution-NonCommercialShareAlike-3.0 License, which permits use, distribution and reproduction for non-commercial purposes, provided the original is properly cited and derivative works building on this content are distributed under the same license. 